\title{
Determination of gas pressure in voids in epoxy casting using an ultrasonic measuring technique
}

\section{Larsen, Esben; Petersen, C. Bak; Henriksen, Mogens}

Published in:

I E E E International Symposium on Electrical Insulation. Conference Record

Link to article, DOI:

10.1109/ELINSL.1990.109785

Publication date:

1990

Document Version

Publisher's PDF, also known as Version of record

Link back to DTU Orbit

Citation (APA):

Larsen, E., Petersen, C. B., \& Henriksen, M. (1990). Determination of gas pressure in voids in epoxy casting using an ultrasonic measuring technique. I E E E International Symposium on Electrical Insulation. Conference Record, 410-413. https://doi.org/10.1109/ELINSL.1990.109785

\section{General rights}

Copyright and moral rights for the publications made accessible in the public portal are retained by the authors and/or other copyright owners and it is a condition of accessing publications that users recognise and abide by the legal requirements associated with these rights.

- Users may download and print one copy of any publication from the public portal for the purpose of private study or research.

- You may not further distribute the material or use it for any profit-making activity or commercial gain

- You may freely distribute the URL identifying the publication in the public portal 


\title{
DETERMINATION OF GAS PRESSURE IN VOIDS \\ IN EPOXY CASTING USING AN ULTRASONIC MEASURING TECHNIQUE.
}

\author{
E. Larsen \& C. Bak Petersen \& M. Henriksen \\ Electric Power Engineering Department, Technical University of Denmark \\ Building 325 \\ DK-2800 Lyngby, Denmark
}

\begin{abstract}
The gas pressure in voids is one of the parameters for determination of the inception voltage for partial discharges in said voids in high voltage epoxy castings.

Seen as the initial step to obtain absolute void gas pressure in closed voids, results of measurements performed on a large open void, where pressure can be controlled from the outside, are compared to the theory of transmission of ultrasound. The results verify the theory, that the attenuation of transmitted ultrasonic signals through a void depends on the gas pressure inside the void.
\end{abstract}

The results are based on the use of the measuring technique Time Delay Spectrometry. This method gives a sufficiently high S/N-ratio in order to detect the transmitted part of the ultrasonic signal, which has travelled through the void. Because of difference in density and velocity of ultrasonic signals for the materials involved, that part of the signal is dependent of the pressure inside the void.

Further steps to measure the pressure inside closed voids are shortly discussed.

\section{Introduction}

The long term aim is to develop a method to determine pressure inside naturally moulded voids in high voltage spacers as well as inside artificially moulded voids, in which degradation is known from laboratory testing under high voltage.

For determination of pressure inside closed voids, the theory of transmission of ultrasound is introduced. By use of this theory, a simple model is developed for calculation of the attenuation of ultrasonic signals through such a system consisting of an epoxy casting including a void.
Because of an extremely high attenuation for ultrasonic signals through a void, a special acoustic measuring technique [1] is used for this purpose.

Results from a test setup are shown to verify the model introduced.

\section{Transmission of Ultrasound Through Materials.}

The transmission of ultrasound through different media and especially transitions between materials depends on the specific acoustic impedance for a given medium. The specific acoustic impedance is defined by:

$$
\mathrm{Z}=\sqrt{\mathrm{E} \cdot \rho}=\rho \cdot \mathrm{C}
$$

where $\rho(\mathrm{p}, \mathrm{T}): \quad$ The density of the medium

$c(T)$ : The velocity of sound in the medium

$\mathrm{E}(\mathrm{p}, \mathrm{T})$ : The modulus of elasticity

p: $\quad$ Pressure.

T: Absolute temperature.

For calculation of the attenuation of ultrasonic signals in transitions from one medium to another, a similar theory as for travelling electrical waves can be used. From this theory the transmission coefficient can be calculated as:

$$
C_{t}=\frac{2 * Z_{2}}{Z_{1}+Z_{2}}
$$

where the specific acoustic impedance of the material the ultrasonic signal comes from is $Z_{1}$ and the material the signal transits to is $\mathrm{Z}_{2}$.

By multiplication of the transmission coefficients for all the transitions between different media, the total attenuation through the specimen can be found. The model does not consider the attenuation of the ultrasonic signal inside each material, which is why the dimensions of the sample or void have no influence on the model. 


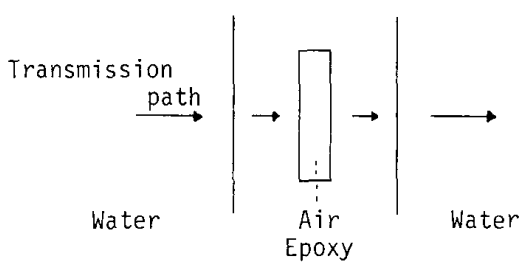

Fig. 1. Sketch of a test sample with an ideal void.

For a system consisting of epoxy with a void all immersed in water, Fig. 1, the signal attenuation depending on the void gas pressure can be determined by use of the theory described above. The dependency of the physical parameters on pressure and temperature can be found in ordinary physical tables.

\begin{tabular}{|c|c|c|c|c|c|}
\hline Attenuation dB & 1 Bar & 2 Bar & 3 Bar & 4 Bar & 5 Bar \\
\hline $20^{\circ} \mathrm{C}$ & 65.2 & 59.1 & 55.7 & 53.2 & 51.2 \\
\hline $50^{\circ} \mathrm{C}$ & 65.5 & 59.5 & 56.0 & 53.5 & 51.6 \\
\hline
\end{tabular}

Fig. 2. Attenuation of ultrasonic signals through a test sample of epoxy including a void.

As seen in fig 2. the signal attenuation is very dependent on the pressure while it is usually acceptable to ignore the temperature dependency.

\section{Time Delay Spectrometry.}

In practise, for determination of the pressure inside voids, a rather new measuring technique called Time Delay Spectrometry is used. Because of different sound velocities inside the

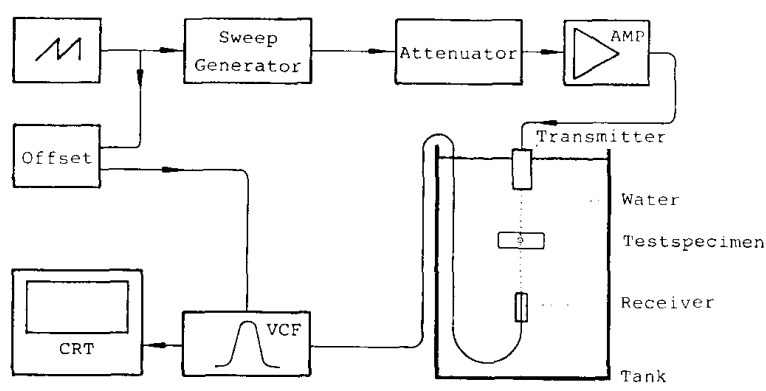

Fig. 3. Test Setup. materials, it is possible to select the part of the signal which has been transmitted through the void. A test signal, generated by a sweep generator, is amplified and fed to a focusing transducer. The frequency of the test signal is linearly increased. The transmitted signal received at the hydrophone is fed to a spectrum analyzer via a variable filter, which normally consists of a fixed filter with an adjustable bandwidth and a mixer circuit.

The signal fed to the focusing transducer, fig. 4, is a signal with a constant amplitude but with a linearly increased frequency in the range of $1-10 \mathrm{MHz}$.
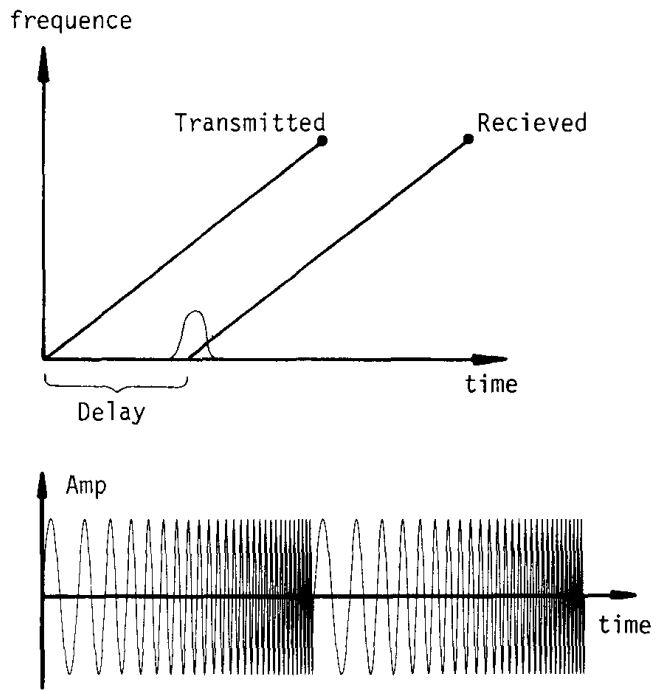

Fig. 4. Linearly increased signal.

As the signal through the materials is delayed corresponding to the limited velocity of ultrasound, the receiving circuit with an adjustable filter may be adjusted corresponding to the transmission time directly through the materials including the void. Other signal paths, for example signals reflected on the tank walls, will usually mean longer transmission times. These signals will be highly attenuated by the filter in the receiving circuit. In that way a sufficiently high $\mathrm{S} / \mathrm{N}$-ratio is obtained in order to discriminate the signal path directly through the void.

Because the pressure dependent signals are attenuated more than $50 \mathrm{~dB}$, it is necessary to very optimise accurately the positions for the transducers as well as the analyzing parameters. For that reason the test setup includes two 3 -axis motion systems (the resolution is less than $5 \mu \mathrm{m}$ on each axis) to which the receiver and the test sample, respectively, are attached whereas the transmitter is fixed. 


\section{Measurements.}

The model described above has been verified on an open test sample where the internal pressure can be controlled from the outside, fig. 5 , in the range of 0.25 to 6 Bar absolute.

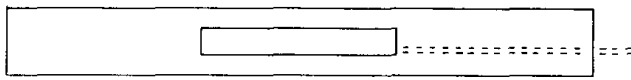

Thickness of the epoxy plate $=6.5 \mathrm{~mm}$. Size of the void $=2 * 20 \mathrm{~mm}$ cylindrical. A needle is used as a channel to the void.

Fig. 5. Open test sample.

The test results are shown in fig. 6 where they are compared to the theoretical results. The model used was, for comparative reasons, adjusted in order to take into account the real sound velocity parameters in the water tank and the density parameters of the epoxy plate valid for the test setup.

As seen in figure 5, a very good correlation was found. The deviation found for pressures below atmospheric pressure was caused by a small $\mathrm{S} / \mathrm{N}$-ratio for these highly attenuated signals.

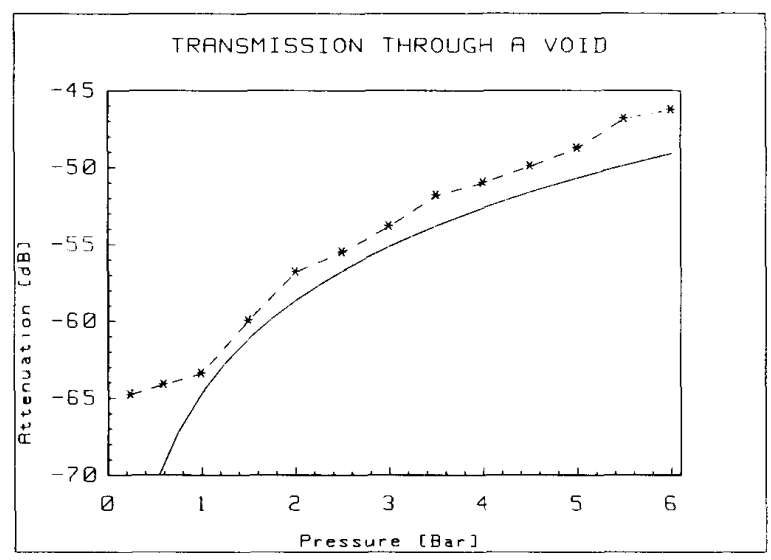

Fig. 6. Comparison of theoretical and measured attenuation of an ultrasonic signal through a void. theoretically, measured

The focusing transducer unfortunately set the limit for the transmitted power to be used because a further increase of the supplied power would reduce the life time of, or in worst case, damage the transducer.
It should be realized that the model does not include the attenuation of the ultrasound by transmission through a material but just attenuations from the transitions. For signal transmission through the plate, but not through the void, (i.e. water-epoxy-water), the signal attenuation is calculated to about $1 \mathrm{~dB}$ while experimentally the attenuation is found to be more than $1.5 \mathrm{~dB} / \mathrm{mm}$ epoxy. The finish of the samples surfaces (polish) seems to have just a minor influence on the signal attenuation.

As a spin off, the method has shown an extremely high sensitivity for detection of defects in solid materials [2]. This facility on the other hand is necessary for use of this test method on non transparent materials in order to locate the void.

\section{Conclusion.}

The presented results verify a simple model for the attenuation of ultrasonic signals through an epoxy specimen including a void. As the attenuation depends on the density and the velocity of sound for the different media, a highly dependance of gas pressure was found theoretically as well as experimentally.

As the pressure dependant signals are attenuated more than $50 \mathrm{~dB}$, a special measuring technique, Time Delay Spectrometry, must be used to obtain the necessary $\mathrm{S} / \mathrm{N}$-ratio. Considerable demands must be made to the resolution of the physical movements of the transducers and the test specimen as well as the parameters for the analyzing circuits.

Further work is expected to show some limitations of the measuring technique. First of all, the size and shape of the voids may be expected to give problems, because the focusing spot of the ultrasonic signal from the focusing transducer is limited to about $1 \mathrm{~mm}$ in diameter. Secondly a signal path just beside the void will have a transmission delay nearly the same as the direct path through the void.

For determination of gas pressure in natural defects in spacers, a later calibration in that same void must be expected. In order to make an absolute pressure determination in a void, that void, after the first pressure measurement, must be cut out of the spacer and be opened to introduce a controlled pressure exposure. By comparison of the first measurement with later measurements with different pressures, the initial pressure in the void can be determined. In the long term, the knowledge of these secondary influences will show if this calibration will still be necessary. 
The authors wish to thank the Electric Power Research Institute for partly funding the work under contract RP2669-1. The authors also wish to thank Vald. Trane og Hustru Elisa Trane's Foundation for partly funding the test setup.
[1] R.C. Heyser, Acoustical Measurement by Time Delay Spectrometry JAES, Vol. 15, no. 4, 1967.

[2] E. Larsen \& C. Bak Petersen, Detection of Defects in Solid Materials using an Ultrasonic Measuring Technique NORD-IS 90, June 1990, Lyngby, Denmark (to be published). 